\title{
SUR LES NÉMATODES PARASITES DE RONGEURS DE MALAISIE
}

\author{
II. Les Trichostrongyloidea. \\ C. K. OW YANG*, M.-C. DURETTE-DESSET** et M. OHBAYASHI***
}

\begin{abstract}
RÉSUMÉ. Vingt-deux espèces de Trichostrongyloïdes parasites de Rongeurs de Malaisie ont été décrites en 1967 dans une thèse non publiée. Quatre de ces espèces ont déjà été redécrites, parfois avec, parfois sans référence à la thèse. Les 18 espèces restantes sont redécrites en utilisant les documents fournis dans la thèse et certains compléments morphologiques (en particulier le synlophe) résultant de l'étude des paratypes. Elles sont reclassées selon les critères de la classification actuelle.

Quatre genres sont créés :

Brevistriatinae :

- Macrostrongylus n. gen. est caractérisé par une bourse caudale de type Calypsostrongylus et l'absence de synlophe.

Nippostrongylinae :

- Malaistrongylus $\mathrm{n}$. gen. est caractérisé par un synlophe de type Heligmonoides, mais avec un plus grand nombre d'arêtes, et par la fusion des côtes 4 et 5 de la bourse caudale.

- Rattustrongylus n. gen. est caractérisé par des arêtes dorsales gauches petites et sub-égales et un nombre d'arêtes inférieur à 20 .

- Sabanema n. gen. est caractérisé par des arêtes dorsales gauches petites et sub-égales et un nombre d'arêtes supérieur à 30 .
\end{abstract}

\section{Studies on the Nematode parasites of Malaysian rodents. II. The Trichostrongyloidea.}

SUMMARY. Many trichostrongyloid species parasitizing rodents in Malaisia were described in 1967 in a thesis that was never published. Some of these species have since been redescribed sometimes with, sometimes without reference to the thesis. The remaining species are redescribed using information given in the thesis and certain additional morphological data (in particular, the synlophe) taken from study of the paratypes. The species are reclassified according to criteria established in the most recent classification. The following genera are proposed :

Brevistriatinae :

- Macrostrongylus n. gen. characterized by a caudal bursa of Calypsostrongylus type and absence of synlophe.

* Institute for Medical Research, Division of Parasitology, Jalan Pahang, Kuala-Lumpur 02-14, Malaysia.

"* Muséum National d'Histoire Naturelle, Laboratoire de Zoologie-Vers, associé au CNRS, 61 rue Buffon, F 75231 Paris Cedex 05. Japan.

*** Faculty of Veterinary Medicine, Department of Parasitology, Hokkaido University, Sapporo 060,

Accepté le 18 janvier 1983 . 
Nippostrongylinae :

- Malaistrongylus n. gen. characterized by a synlophe of Heligmonoides type but with a larger number of ridges and by the fusion of rays 4 and 5 in the caudal bursa.

- Rattus strongylus $\mathrm{n}$. gen. characterized by small, subequal dorsal left ridges and a total number of ridges less than 20 .

- Sabanema n. gen. characterized by small subequal dorsal left ridges and a total number of ridges greater than 30 .

The species under consideration are the following :

Hepatojarakus malayae Yeh, 1955 ; Pithecostrongylus bicapitatus n. sp. (= P. bicapitatus Ow Yang, 1967, in litt) ; Macrostrongylus ratti n. gen., n. sp. (= Macrostrongylus ratti Ow Yang, 1967, in litt.) ; Calypsostrongylus malayensis Durette-Desset, 1976 (= Brevistriata malayensis Ow Yang, 1967, in litt) ; Fissicauda callosciuri (Supperer et Kutzer, 1964) ; Fissicauda brevispicula n. sp. (= Brevistriata brevispicula Ow Yang, 1967, in litt.) ; Nippostrongylus brasiliensis (Travassos, 1914) ; Orientostrongylus tenorai Durette-Desset, 1970 (= Longistriata selangora Ow Yang, 1967, in litt.) ; O. krishnansamyi Durette-Desset et Lim-Boo-Liat, 1974 (= Longistriata malaccae Ow Yang, 1967, in litt.); Heligmonoides bulbosus n. sp. (= Heligmonina (Heligmonoides) bulbosa Ow Yang, 1967, in litt.) ; Heligmonoides lanceolatus n. sp. (= Heligmonina (Heligmonoides) lanceolata Ow Yang 1967, in litt.); Malaistrongylus odontospicularis n. gen., n. sp. (= Malaistrongylus odontospicularis Ow Yang, 1967, in litt.) ; Paraheligmonelloides triangulus $\mathrm{n}$. sp. (= Longistriata triangulum Ow Yang, 1967, in litt.) ; P. annandalei n. sp. (= Longistriata annandalei Ow Yang, 1967, in litt.) ; P. rajah n. sp. (= Heligmonina (Heligmonoides) rajah Ow Yang, 1967, in litt.) ; Rattustrongylus odontoconus n. gen., n. sp. (= Longistriata odontocona Ow Yang, 1967, in litt.) ; $R$. rotundoconus n. sp. (= Longistriata rotundocona Ow Yang, 1967, in litt.) ; Sabanema sabana n. gen., n. sp. (= Longistriata sabana Ow Yang, 1967, in litt.) ; S. kepongi n. sp. (= Longistriata kepongi Ow Yang, 1967, in litt.); S. macrovulva n. sp. (= Longistriata macrovulva Ow Yang, 1967, in litt.) ; S. montana n. sp. (= Heligmonina (Neoheligmonina) montana Ow Yang, 1967, in litt.) ; S. longispicularis n. sp. (= Pseudoheligmostrongylus longispicularis Ow Yang, 1967, in litt.).

\section{Introduction}

L'un d'entre nous (Ow Yang, 1967) a décrit de nombreuses nouvelles espèces de Nématodes Trichostrongyloïdes, essentiellement parasites de Muridae en Malaisie.

Dans ce travail, qui faisait partie d'une thèse pour l'obtention d'un $\mathrm{Ph}$. D., seule la première partie (Rhabdiasidae, Trichuridae et Oxyuridae) a été publiée (Ow Yang, 1971). La seconde partie (Trichostrongyloidea) n'a pas été publiée et la thèse elle-même n'a pas été mise en vente, ni distribuée. Il en est résulté, et il en résulte encore, de nombreuses difficultés. L'une d'entre nous (Durette-Desset), en 1970, puis en 1974 (en collaboration avec Lim-Boo-Liat), a redécrit deux des espèces précédentes sous des noms différents et, en 1976, deux autres espèces sous le même nom que celui qui est utilisé dans la thèse. Par ailleurs, l'un d'entre nous (Ohbayashi) travaille actuellement sur des espèces parasites de Muridae de Thailande, dont une au moins correspond aux formes décrites par Ow Yang en Malaisie. Il devient donc urgent de redonner une brève description et une illustration suffisantes des espèces en cause, et de les publier selon les critères de publication du Code International de Nomenclature (articles 7-8-9). Les types de chaque espèce sont déposés dans les Collections de la 
London School of Hygiene and Tropical Medicine et ont été étiquetés sous la dénomination figurant dans la thèse de 1967.

Du point de vue nomenclature, 3 cas se présentent :

a) L'espèce a été redécrite sans référence à la thèse. Il s'agit donc d'une dénomination valide. Il est intéressant cependant d'indiquer quel est le taxon non valide qui lui correspond dans la thèse.

Ex. : Orientostrongylus tenorai Durette-Desset, 1970

(= Longistriata selangora Ow Yang, 1967, in litt.)

b) L'espèce a été redécrite avec référence à la thèse et le nom spécifique d'origine a été conservé et est donc déjà validé.

Ex. : Calypsostrongylus malayensis Ow Yang in Durette-Desset, 1976

(= Brevistriata malayensis Ow Yang, 1967, in litt.)

c) L'espèce n'a pas encore été redécrite. Nous validons donc ici le nom spécifique proposé en 1967.

Ex. : Pithecostrongylus bicapitatus n. sp.

(= Pithecostrongylus bicapitatus Ow-Yang, 1967, in litt.)

La systématique des Nématodes Trichostrongyloïdes ayant beaucoup changé depuis 1967, les espèces sont reclassées suivant les données actuelles et plusieurs genres nouveaux doivent ètre créés.

\section{I - Molineidae - Molineinae}

1 - Genre Hepatojarakus Yeh, 1955

- Hepatojarakus malayae Yeh, 1955

Hôtes et origine géographique : - Rattus rattus jarak (Matériel type), $R$. exulans concola, $R$. annandalei, $R$. alticola, $R$. argentiventer, $R$. bowersi, $R$. canus, $R$. cremoriventer, $R$. rattus diardi, $R$. exulans, $R$. tiomanicus, $R$. rajah, $R$. whiteheadi, de Malaisie (voir Ow Yang, 1967 ; Schacher et Cheong, 1960 ; Singh et Cheong, 1971). - Tupaia tana, Aeromys thomasi, de Borneo (voir Durette-Desset et Chabaud, 1975).

2 - Genre Pithecostrongylus Lubimov, 1930

- Pithecostrongylus bicapitatus n. sp.

(= Pithecostrongylus bicapitatus Ow Yang, 1967 in litt.)

Hôtes et origine géographique: Rattus cremoriventer (Matériel type),

R. tiomanicus, de Malaisie.

Description (fig. 1, $A$ à $C$ )

- Synlophe: 10 arêtes cuticulaires au niveau du pore excréteur.

- Principales mensurations :

Mâle : longueur : 3,25 (3,12-3,38) mm sur $92(85-99) \mu \mathrm{m}$. Spicules : $156(153-158) \mu \mathrm{m}$. Gubernaculum : $50(49-51 \mu \mathrm{m})$.

Femelle : longueur : $3,16 \mathrm{~mm}$ sur $57 \mu \mathrm{m}$. Vulve à $774 \mu \mathrm{m}$ de l'extrémité caudale. Eufs hauts de $55(54-56) \mu \mathrm{m}$ sur $32(22-32) \mu \mathrm{m}$. Queue : $85 \mu \mathrm{m}$. 


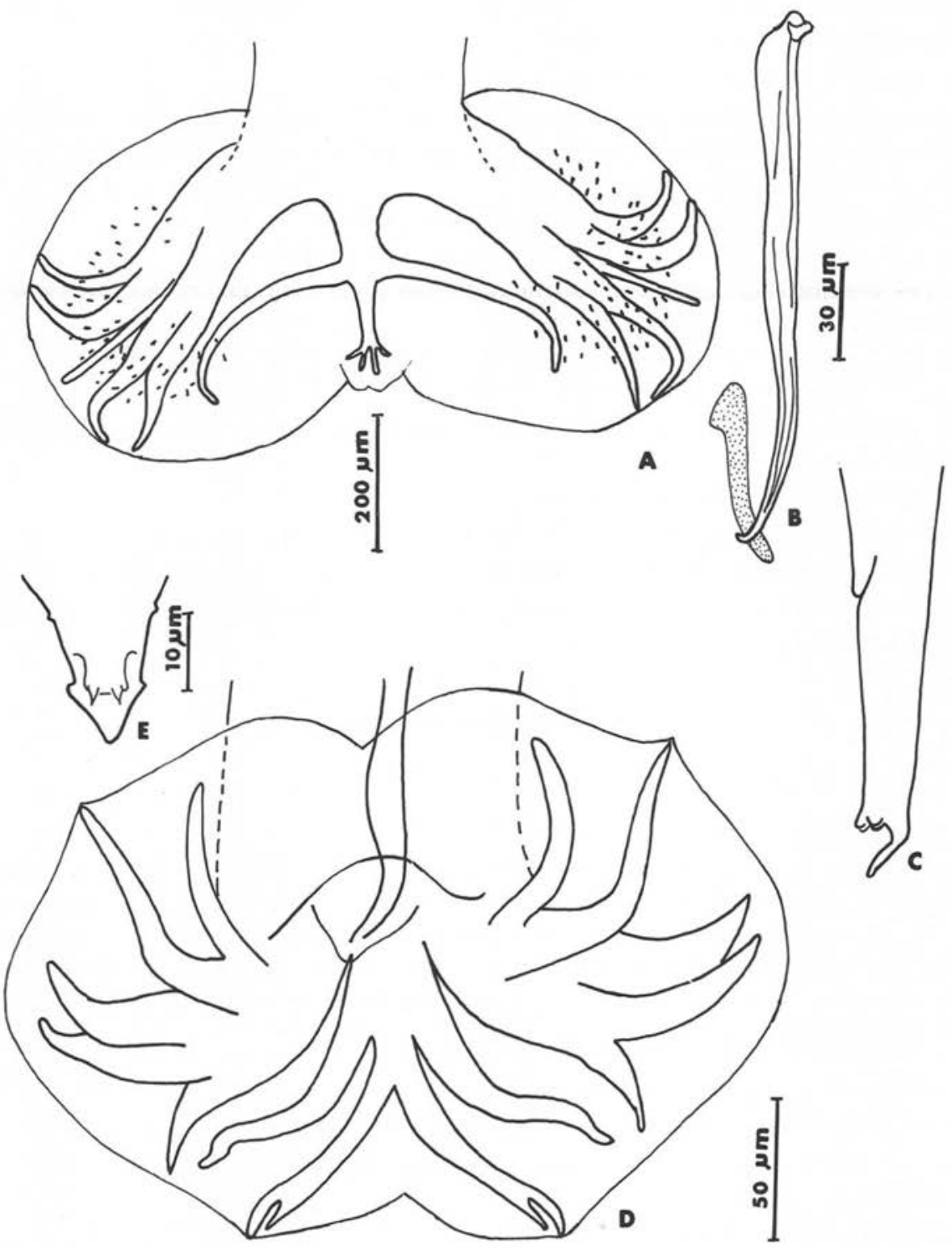

FIG. I. - Pithecostrongylus bicapitatus n. sp.

A, $\hat{\delta}$, bourse caudale, vue ventrale. B, $\hat{\delta}$, spicule droit et gubernaculum, vue latérale droite. $\mathrm{C}$,, , extrémité postérieure, vue latérale gauche. Fissicauda brevispicula n. sp. D, $\delta$, bourse caudale, vue ventrale. E, cône génital, vue dorsale.

(D'après Ow Yang, 1967, in litt.). Pour se conformer à l'usage, la bourse caudale a été dessinée en vue ventrale, et, de ce fait, le dessin original a été retourné. 


\section{Discussion}

Bien que l'orientation de la pointe des arêtes ne soit pas connue, les spécimens ci-dessus peuvent être rangés dans le genre Pithecostrongylus, dont ils possèdent les autres caractères, à savoir : extrémités des côtes 2 et 3 formant une " pince " antérieure et des côtes 5 et 6 formant une " pince " postérieure. Épine caudale chez la femelle.

Parmi les espèces du genre, $P$. serratus (Ow Yang, 1965), parasite de Sciuridés de Malaisie, paraît l'espèce la plus proche ; en particulier, l'extrémité des côtes 4 se trouve équidistante de celle des 3 et 5 , et la côte dorsale est bien développée.

Les spécimens ci-dessus s'en éloignent par des côtes 4 très grêles, des côtes 8 naissant à la moitié de la dorsale et des spicules sans indentations.

\section{II — Heligmonellidae}

\section{A - Brevistriatinae}

1 - Genre Macrostrongyhus gen. nov.

(= Macrostrongylus Ow Yang, 1967, in litt.)

- Macrostrongylus ratti $\mathrm{n}$. sp.

(= Macrostrongylus ratti Ow Yang, 1967, in litt.)

Hôtes et origine géographique: Rattus sabanus (Matériel type), R. rattus diardi, R. rajah, R. whiteheadi, de Malaisie.

Description (fig. 2).

- Synlophe: absent.

- Principales mensurations :

Mâle : Longueur : 13,2 (12,3-15,2) mm sur 226 (185-270) $\mu \mathrm{m}$. Vésicule céphalique : $50(46-56) \mu \mathrm{m}$ sur $72(68-83) \mu \mathrm{m}$. Anneau nerveux et pore excréteur à $262(227-320) \mu \mathrm{m}$ et $758(675-930) \mu \mathrm{m}$ de l'apex. Esophage : $461(419-497) \mu \mathrm{m}$. Spicules : 1,69 (1,561,82) mm. Gubernaculum : 83 (68-102) $\mu \mathrm{m}$.

Femelle : Longueur : 26,9 (23-29) mm sur 517 (454-604) $\mu \mathrm{m}$. Vésicule céphalique : 57 (46-68) $\mu \mathrm{m}$ sur 105 (94-119) $\mu \mathrm{m}$. Anneau nerveux et pore excréteur à $303(284-320) \mu \mathrm{m}$ et $760(639-846) \mu \mathrm{m}$ de l'apex. Esophage : $551(511-582) \mu \mathrm{m}$. Vulve à $231(185-236) \mu \mathrm{m}$ de l'extrémité caudale. Nombreux œufs hauts de 68 (65-73) $\mu \mathrm{m}$ sur 41 (39-41) $\mu \mathrm{m}$.

\section{Discussion}

Les parasites ci-dessus possèdent un ensemble de caractères originaux chez un Trichostrongyloïde : très grande taille, absence de synlophe ; pore excréteur s'ouvrant très postérieurement ; allongement considérable du vestibule.

Cependant, les caractères bursaux (bourse caudale de type 2-2-1; côte dorsale bien développée) évoquent des Brevistriatinae comme Calypsostrongylus longipene Durette-Desset et Chabaud, 1967, parasite d'un Écureuil volant indochinois, ou Cordicauda trichysi, parasite de Trichys lipura en Malaisie. 

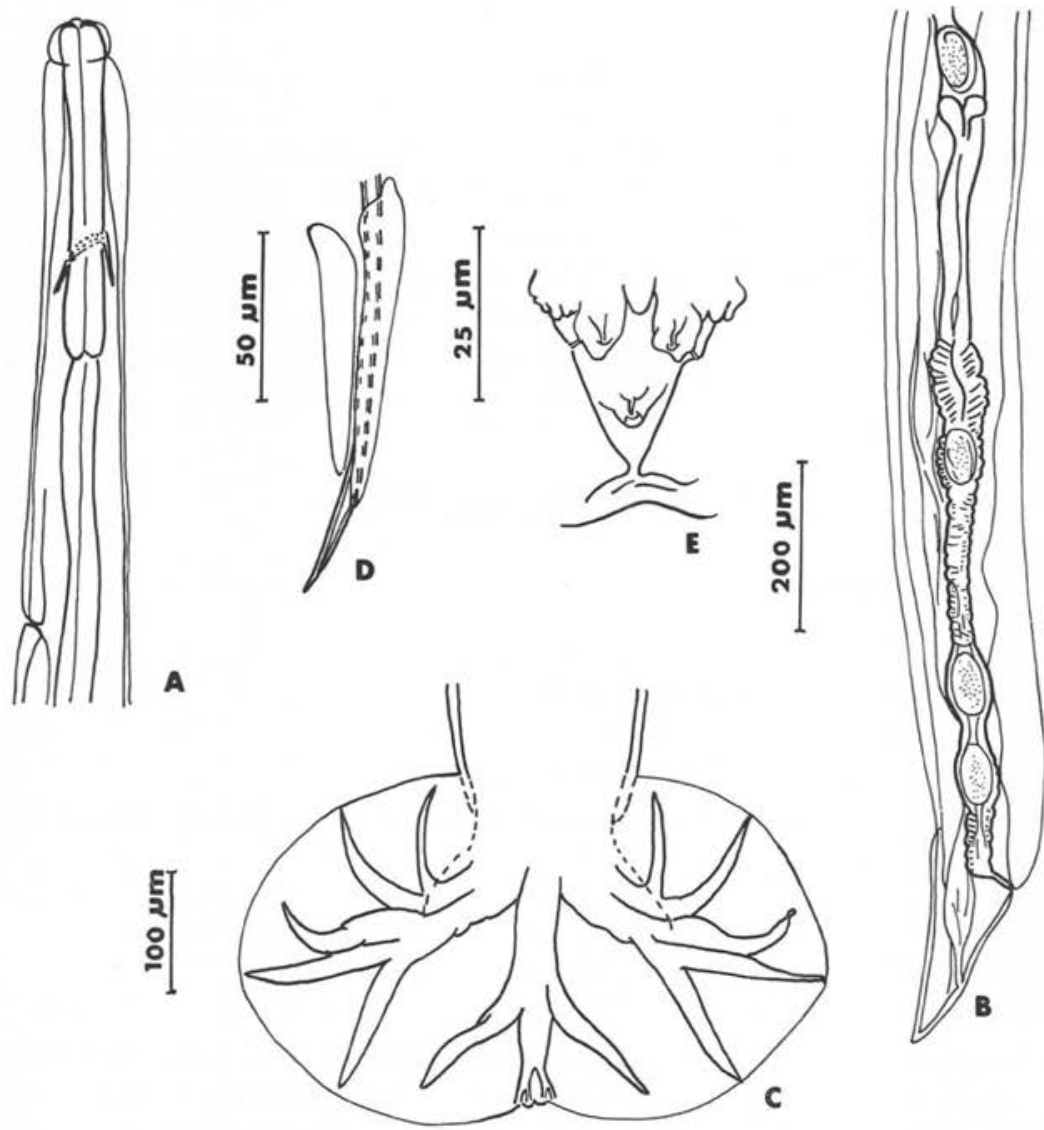

FIG. 2. - Macrostrongylus ratti n. gen., n. sp. A, extrémité antérieure, vue latérale gauche. B, $q$, extrémité postérieure, vue latérale droite. C, $\widehat{\sigma}$, bourse caudale, vue ventrale (dessin original retourné). D, $\widehat{\delta}$, telamon et gubernaculum, vue latérale gauche. E, cône génital, vue ventrale. (D’après Ow Yang, 1967 , in litt.)

Il nous paraît donc possible de classer ce genre dans les Brevistriatinae, en considérant que le genre le plus proche est Cordicauda (Durette-Desset, 1971,) car, en plus des caractères de la bourse caudale, certaines espèces de ce genre présentent un allongement important du vestibule.

Définition: Genre Macrostrongylus n. gen. : Heligmonellidae, Brevistriatinae : Très grande taille (dépassant le centimètre). Absence de synlophe. Bourse caudale de type 2-2-1, avec côte dorsale bien développée.

Vestibule de la femelle très allongé.

Parasite de Rongeurs Muridae orientaux.

Espèce type unique: Macrostrongylus ratti n. gen., n. sp. 
La majorité des Brevistriatinae sont parasites de Rongeurs archaïques, mais nous interprétons la présence de ce genre chez les Muridae comme un phénomène de capture à partir de genres proches de Cordicauda, par exemple.

2 - Genre Calypsostrongylus Schmidt, Myers et Kuntz, 1967

- Calypsostrongylus malayensis Durette-Desset, 1976.

(= Brevistriata malayensis Ow Yang, 1967, in litt.)

Hôtes et origine géographique: Rattus canus (Matériel type), $R$. annandalei, R. argentiventer, $R$. rattus diardi, $R$. rajah, $R$. sabanus, de Malaisie (voir Ow Yang, 1967) ; Aeromys tephromelas, Callosciurus caniceps, de Malaisie (voir Durette-Desset, 1976).

L'espèce est figurée et redécrite par Durette-Desset ,1976.

3 - Genre Fissicauda Durette-Desset et Krishnasamy, 1976.

a) Fissicauda callosciuri (Supperer et Kutzer, 1964).

Hôtes et origine géographique:-Callosciurus prevosti (Zoo d'Autriche) (voir Supperer et Kutzer, 1964). - Différents Écureuils et, en particulier, Callosciurus notatus, Rattus canus et $R$. mulleri, de Malaisie (voir Ow Yang, 1967); C. notatus de Malaisie (voir Durette-Desset et Krishnasamy, 1976).

L'espèce ayant été décrite à différentes reprises dans des travaux normalement publiés n'est pas reconsidérée ici.

b) Fissicauda brevispicula n. sp.

(= Brevistriata brevispicula Ow Yang, 1967, in litt.)

Hôtes et origine géographique: Rattus sabanus (Matériel type), R. tiomanicus R. whiteheadi, de Malaisie.

L'espèce a été citée sans redescription par Durette-Desset et Krishnasamy, 1976. Description ( $f i g .1, D-E$ ) - La femelle est inconnue.

- Synlophe: au niveau des deirides, 16 arêtes interrompues.

- Principales mensurations:

Mâle : longueur : $6,68 \mathrm{~mm}$ sur $121 \mu \mathrm{m}$. Spicules : $568 \mu \mathrm{m}$, à pointe simple. Gubernaculum : $120 \mu \mathrm{m}$.

Discussion

Parmi les Fissicauda, les spécimens ci-dessus se distinguent aisément de toutes les autres espèces du genre, car ce sont les seuls à avoir à la fois les 2 caractères suivants : très faible écartement de l'extrémité des côtes 6 et 8 et spicules à pointe unique.

\section{B - Nippostrongylinae}

Toutes les espèces qui suivent présentent les principaux caractères des Nippostrongylinae, à savoir :

— absence de capsule buccale et de dent œsophagienne dorsale ; 
- monodelphie ;

- bourse caudale de type 2-2-1 ;

— axe d'orientation des arêtes incliné à plus de $67^{\circ}$ sur l'axe sagittal.

1) Genre Nippostrongylus Lane, 1923

- Nippostrongylus brasiliensis (Travassos, 1914)

Hôtes et origine géographique: Rongeurs domestiques : Rattus rattus, $R$. norvegicus, Mus musculus dans le monde entier. Rattus tiomanicus, $R$. argentiventer, $R$. rattus diardi de Malaisie (voir Ow Yang, 1967).

L'espèce a été décrite selon les critères modernes par Chabaud et DuretteDesset, 1966.

2 - Genre Orientostrongylus Durette-Desset, 1970

a) Orientostrongylus tenorai Durette-Desset, 1970

(= Longistriata selangora Ow Yang, 1967, in litt.)

Hôtes et origine géographique: - Rattus jalorensis (Matériel type), R. argentiventer, $R$. rattus diardi, $R$. exulans, $R$. mulleri, $R$. whiteheadi, de Malaisie (voir Ow Yang, 1967). - Bandicota bengalensis, de Chine (voir Durette-Desset, 1970). - Mus musculus, d'Afghanistan (voir Tenora, 1969). - Bandicota indica, B. savilei, de Thaillande (voir Ohbayashi et Kamiya, 1980).

L'espèce a été redécrite par Durette-Desset, 1970.

b) Orientostrongylus krishnansamyi Durette-Desset et Lim-Boo-Liat, 1974 (= Longistriata malaccae Ow Yang, 1967, in litt.)

Hôtes et origine géographique: Rattus argentventer (Matériel type), R. exulans, R. rattus diardi, R. jalorensis, R. whiteheadi, de Malaisie (voir Ow Yang, 1967) ; Iomys horsefieldi, de Malaisie (voir Durette-Desset et Lim-Boo-Liat, 1974).

L'espèce a été redécrite par Durette-Desset et Lim-Boo-Liat, 1974.

3 - Genre Heligmonoides Baylis, 1928

a) Heligmonoides bulbosus n. sp.

[= Heligmonina (Heligmonoides) bulbosa Ow Yang, 1967, in litt. $]$

Hôte et origine géographique: Rattus whiteheadi, de Malaisie.

Description (fig. 3, $A$ à $C$, et fig. 11, $A-B$ ).

- Synlophe : 22 (ठ), 23 (ㅇ) arêtes cuticulaires au milieu du corps. Naissance des arêtes médianes sur le bord postérieur de la vésicule céphalique. Les latérales naissent à différents niveaux le long des champs. Disparition des arêtes au niveau de la bourse caudale chez le ồ et du sphincter chez la q. Axe d'orientation des arêtes de la ligne ventrale-droite vers la ligne gauche. 2 à 4 arêtes gauches hypertrophiées. Dans la partie postérieure du corps, toutes les arêtes sont subégales.

- Principales mensurations :

Mâle : Longueur : 1,49 (1,47-1,51) mm sur $64 \mu \mathrm{m}$ de large. Spicules : $96(92-100) \mu \mathrm{m}$. Gubernaculum : $40-45 \mu \mathrm{m}$. 
Femelle : Longueur : $2,3(2,18-3,24) \mathrm{mm}$ sur $64 \mu \mathrm{m}$ de large. Vulve à $110(102-122) \mu \mathrm{m}$ de l'extrémité caudale. 8 œufs hauts de 68 (51-77) $\mu \mathrm{m}$ sur 30 (27-36) $\mu \mathrm{m}$ de large. Queue : 56 (54-58) $\mu \mathrm{m}$.

\section{Discussion}

Par ses caractères bursaux et son synlophe, l'espèce peut aisément être classée dans le genre Heligmonoides.

L'espèce la plus proche est $H$. parvulus (Durette-Desset, 1966), parasite de Muridae éthiopiens. Les 2 espèces sont de même taille, avec un nombre équivalent de crêtes cuticulaires et une côte dorsale courte divisée dans sa moitié antérieure. Mais, chez les spécimens malais, les spicules sont presque trois fois plus petits et il existe seulement 2 fortes arêtes gauches au lieu de 4 .

b) Heligmonoides lanceolatus n. sp.

[= Heligmonina (Heligmonoides) lanceolata Ow Yang, 1967, in litt.]

Hôte et origine géographique: Rattus rajah, de Malaisie.

Description (fig. 3, D à F).

- Synlophe: 18 ( $\left.0^{\wedge}\right)$ arêtes cuticulaires au niveau de l'œsophage.

- Principales mensurations:

Mâle : Longueur : $1,73(1,67-1,82) \mathrm{mm}$ sur $73(71-78) \mu \mathrm{m}$. Spicules : $458(445-476) \mu \mathrm{m}$. Gubernaculum : $102 \mu \mathrm{m}$.

Femelle : Longueur : 2,39 (2,00-2,78) mm sur 103 (99-107) $\mu \mathrm{m}$. Vulve à $111(85-136) \mu \mathrm{m}$ de l'extrémité caudale. 6 œufs hauts de 50-60 $\mu \mathrm{m}$ sur 32-34 $\mu \mathrm{m}$. Queue : $50 \mu \mathrm{m}$.

\section{Discussion}

Cette espèce nous paraît difficile à classer. La bourse caudale pourrait être celle d'un Heligmonoides ou d'un Paraheligmonelloides, mais la description du synlophe au niveau de l'œsophage, avec 1 forte arête droite et 2 séries opposées de grandes arêtes (dorsales droites et ventrales gauches) ne correspond à aucun synlophe connu. Le synlophe n'étant décrit que sur 5 spécimens mal fixés, et uniquement dans la région de l'œsophage, il ne nous paraît pas actuellement souhaitable de créer un nouveau genre pour cette espèce.

Les analogies étant plus grandes avec la bourse caudale d'Heligmonoides (ventrales fines, côtes 4 nettement plus grandes que les 5, lobe dorsal réduit), nous classons, du moins provisoirement, cette espèce dans le genre Heligmonoides. L'espèce la plus proche paraît être l'espèce type avec des côtes 4 et 5 gauches très épaisses comme chez les spécimens malais. Mais le nombre d'arêtes cuticulaires est de 30 chez H. murina Baylis, 1928, au lieu de 18 chez nos spécimens.

4 - Genre Malaistrongylus n. gen. (= Malaistrongylus Ow Yang, 1967 in litt.)

- Malaistrongylus odontospicularis n. gen., n. sp.

(= Malaistrongylus odontospicularis Ow Yang, 1967, in litt.)

Hôtes et origine géographique:-Rattus sabanus (Matériel type), R. canus, R. rajah, $R$. whiteheadi, Chiropodomys gliroides, de Malaisie. $-R$. surifer, de Thaïlande. 


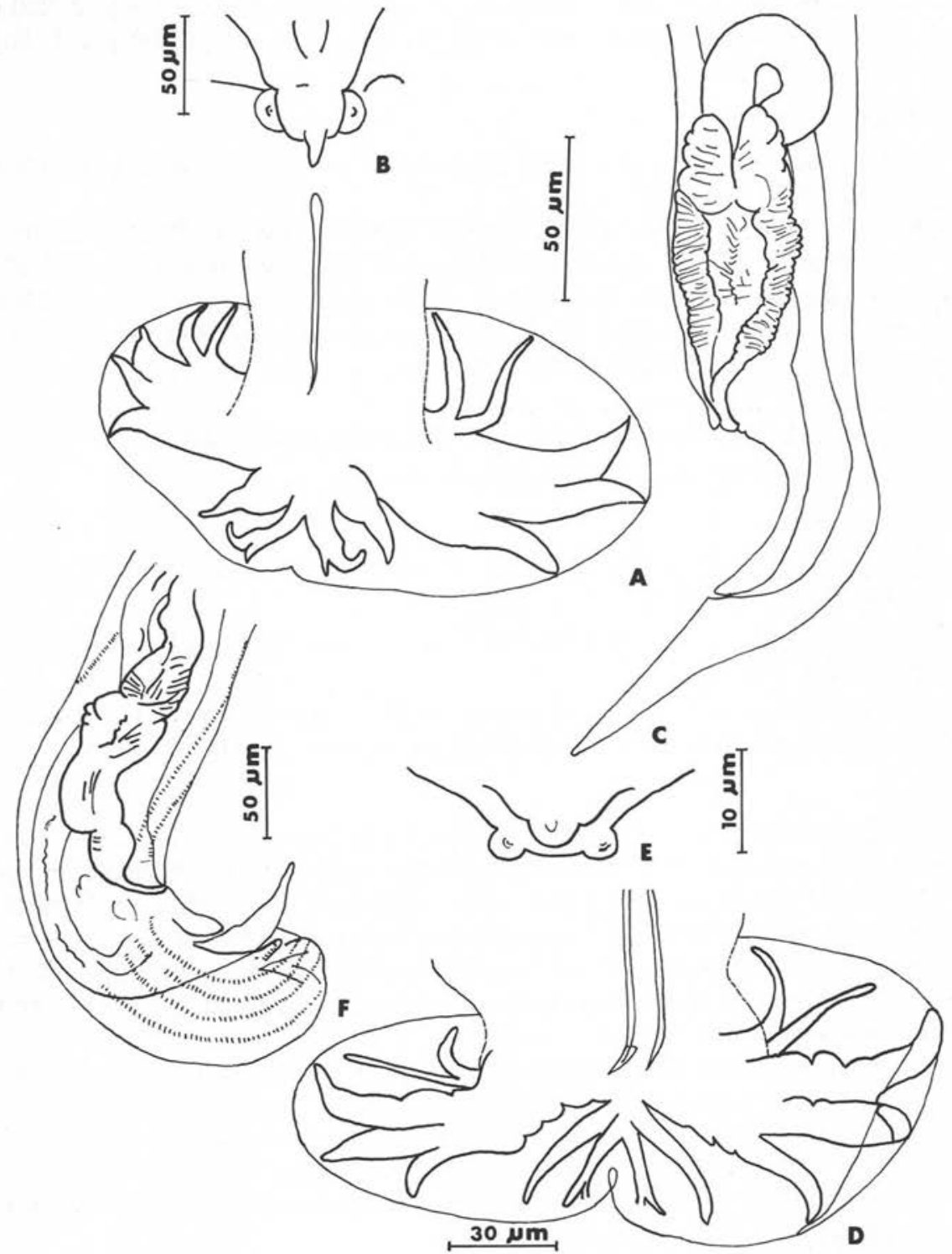

FIG. 3. - Heligmonoides bulbosus n. sp. A, $\hat{0}$, bourse caudale, vue ventrale (dessin original retourné). B, cône génital, vue ventrale. C, ơ, extrémité postérieure, vue latérale gauche. Heligmonoides lanceolatus n. sp. D, $\hat{\delta}$, bourse caudale, vue ventrale (dessin original retourné). E, $\hat{\delta}$, cône génital, vue ventrale. $\mathrm{F}$, $\mathrm{O}$, extrémité postérieure, vue latérale droite.

(D'après Ow Yang, 1967, in litt.). 
Description (fig. 4).

— Synlophe: 32 à 34 arêtes (̂) $), 40$ à 42 arêtes (ㅇ) au milieu du corps. Naissance des arêtes médianes derrière la vésicule céphalique, latérales à différents niveaux le long des champs. Disparition des arêtes au niveau de la bourse caudale chez le $\sigma^{*}$ et de la vulve chez la ․

Axe d'orientation de la ligne ventrale droite vers la ligne gauche, dorsale-gauche.

Arêtes droites et dorsales-gauches plus développées que les autres arêtes. Présence de 2 très fortes dilatations cuticulaires, gauche et ventrale.

\section{- Principales mensurations :}

Mâle : Longueur : 3,68 (3,20-4,33) mm sur $132(92-192) \mu \mathrm{m}$ de large.

Vésicule céphalique : 57 (51-68) $\mu \mathrm{m}$ sur $33(29-37) \mu \mathrm{m}$. Anneau nerveux et pore excréteur à $198(119-249) \mu \mathrm{m}$ et $274(114-334) \mu \mathrm{m}$ de l'apex. Deirides au même niveau. Esophage : $380(334-454) \mu \mathrm{m}$. Spicules : $210(254-327) \mu \mathrm{m}$. Gubernaculum : $22 \mu \mathrm{m}$.

Femelle : Longueur : 4,74 (3,79-5,93) mm sur $163(135-213) \mu \mathrm{m}$ de large.

Vésicule céphalique : $63(54-77) \mu \mathrm{m}$ sur $37(29-44) \mu \mathrm{m}$. Anneau nerveux et pore excréteur à 203 (163-249) $\mu \mathrm{m}$ et 323 (298-362) $\mu \mathrm{m}$ de l'apex. Deirides au même niveau. Esophage : $450(426-476) \mu \mathrm{m}$. Vulve à $197(179-241) \mu \mathrm{m}$ de l'extrémité caudale. Une vingtaine d'œufs hauts de 78 (71-82) $\mu \mathrm{m}$ sur 39 (37-41) $\mu \mathrm{m}$ de large.

Dans le matériel de Thaillande, 26 q sur 46 possèdent un bouchon vulvaire haut de 200 à $250 \mu \mathrm{m}$ en forme de demi-anneau recouvrant la partie ventrale du corps. Cet anneau s'enlève aisément par manipulation, mais sa partie centrale reste enfoncée dans le vagin à la façon d'un clou. Queue : $69(66-67) \mu \mathrm{m}$.

\section{Discussion}

Parmi les genres appartenant aux Nippostrongylinae, Heligmonoides parait être le genre le plus proche : présence d'une dilatation cuticulaire gauche soutenue par des arêtes très fortes et dont le nombre est supérieur à 2, disposition des côtes bursales avec un lobe gauche plus grand, division de la côte dorsale dans sa moitié antérieure.

Malaistrongylus s'éloigne d'Heligmonoides par un nombre plus élevé de crêtes cuticulaires, par l'absence d'arêtes ventrales gauches beaucoup plus fortes que les autres ventrales, et par la fusion des côtes 4 et 5 de la bourse caudale.

Définition: Genre Malaistrongylus n. gen. : Heligmonellidae, Nippostrongylinae. - Synlophe avec nombre d'arêtes cuticulaires supérieur à 30. Arêtes dorsales, gauches et droites les plus développées. Côte dorsale divisée dans sa moitié antérieure. Côtes 4 et 5 fusionnées.

Parasite de Rongeurs Muridae orientaux.

Espèce type unique: Malaistrongylus odontospicularis n. gen. n. sp.

5 - Genre Paraheligmonelloides Fukumoto, Kamiya et Suzuki, 1980

a) Paraheligmonelloides triangulus n. sp.

(= Longistriata triangulum Ow Yang, 1967, in litt.) 


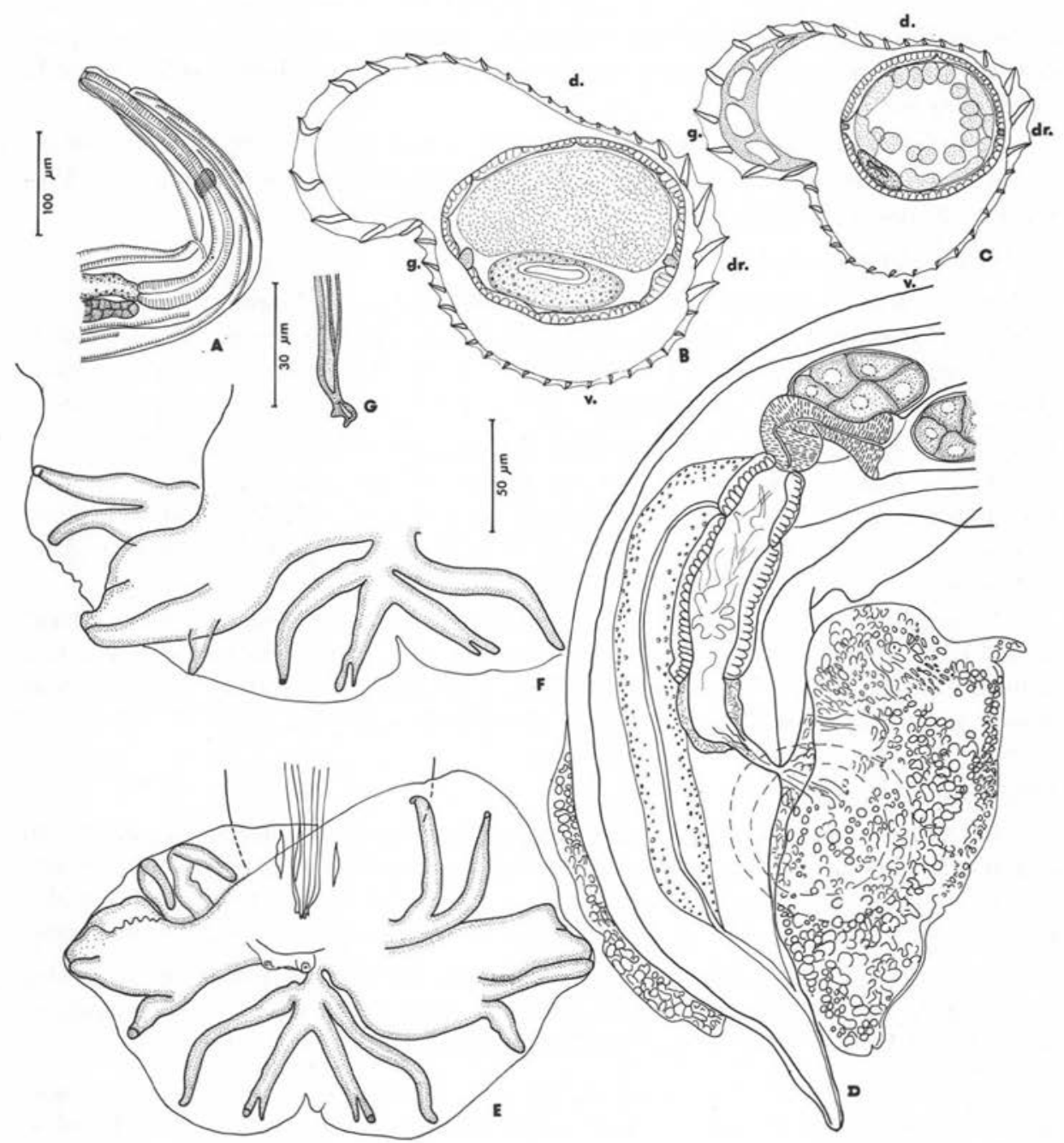

FIG. 4. - Malaistrongylus odontospicularis n. gen., n. sp. A, $\hat{\sigma}$, extrémité antérieure, vue latérale gauche. $\mathrm{B}, \uparrow$, coupe transversale au milieu du corps. C, $\hat{0}, i d$. D, + , extrémité postérieure, vue latérale droite. Le bouchon vulvaire a été légèrement déplacé. $\mathrm{E}, \hat{o}$, bourse caudale, vue ventrale. $F$, autre $\hat{\sigma}$, loble droit étalé, vue ventrale. $G, \hat{\sigma}$, pointe du spicule droit, vue ventrale.

A, éch. : $100 \mu \mathrm{m}$. B à F, éch. : $50 \mu \mathrm{m}$. G, éch. : $30 \mu \mathrm{m}$.

(D'après le matériel de Thaillande. Hôte : Rattus surifer.) 
Hôtes et origine géographique: Rattus sabanus (Matériel type), R. rajah, Chiropodomys gliroides, de Malaisie.

Description (fig. 5, A à D, et fig. 11-C).

- Synlophe: $19(\widehat{\zeta}), 21$ (ㅇ) arêtes au milieu du corps. Arête dorsale gauche plus forte que les autres dorsales.

- Principales mensurations :

Mâle : Longueur : 2,33 (2,16-2,67) mm sur 73 (57-85) $\mu$ m de large. Spicules : 83 (61-85) $\mu \mathrm{m}$, divisés en 3 branches. Gubernaculum : 20 (14-34) $\mu \mathrm{m}$.

Femelle : Longueur : 3,25 (2,96-3,98) $\mathrm{mm}$ sur $78(64-75) \mu \mathrm{m}$ de large. Vulve à 149 (119-179) $\mu \mathrm{m}$ de l'extrémité caudale. 9 œufs hauts de 67 (66-68) $\mu \mathrm{m}$ sur $34(32-37) \mu \mathrm{m}$. Queue : 60 (51-68) $\mu \mathrm{m}$.

\section{Discussion}

La présence simultanée d'un synlophe de type Orientostrongylus et d'une bourse caudale de type Heligmonoides permet de ranger cette espèce dans le genre Paraheligmonoides, connu seulement jusqu'à présent chez un Lagomorphe éthiopien.

Les spécimens malais se distinguent de l'espèce type par 21 arêtes au lieu de 14, par une bourse caudale avec un lobe dorsal bien développé, et par des spicules se terminant par 3 branches soutenues par une membrane.

b) Paraheligmonelloides annandalei n. sp.

(= Longistriata annandalei Ow Yang, 1967, in litt.)

Hôtes et origine géographique: Rattus annandalei (Matériel type), R. mulleri, de Malaisie.

Description (fig. 5, $E$ à $H$, et fig. 11-D).

- Synlophe: 14 (ㅇ) arêtes cuticulaires au milieu du corps. Arête dorsale gauche plus développée que les autres arêtes.

- Principales mensurations :

Mâle : Longueur : 2,28 (1,83-2,64) mm sur 80 (69-92) $\mu \mathrm{m}$. Spicules : $349(326-362) \mu \mathrm{m}$, bifurqués à leur extrémité.

Femelle : Longueur : 2,41 (2,02-2,82) mm sur $75(64-78) \mu \mathrm{m}$. Vulve à 88 (78-102) $\mu \mathrm{m}$ de l'extrémité caudale. 9 œufs hauts de 67 (60-73) sur 31 (27-34) $\mu$ m. Queue : 36 (34-46) $\mu \mathrm{m}$.

\section{Discussion}

Pour les mêmes raisons que pour l'espèce précédente, l'espèce est rangée dans le genre Paraheligmonelloides.

L'espèce la plus proche est l'espèce type $P$. kenyensis Fukumoto et coll., 1980 : le nombre d'arêtes cuticulaires est le même et la disposition des côtes bursales très semblable.

Les spécimens malais s'en différencient par une côte dorsale moins profondément divisée, des côtes 4-5-6 plus épaisses et par des spicules à 2 branches. 

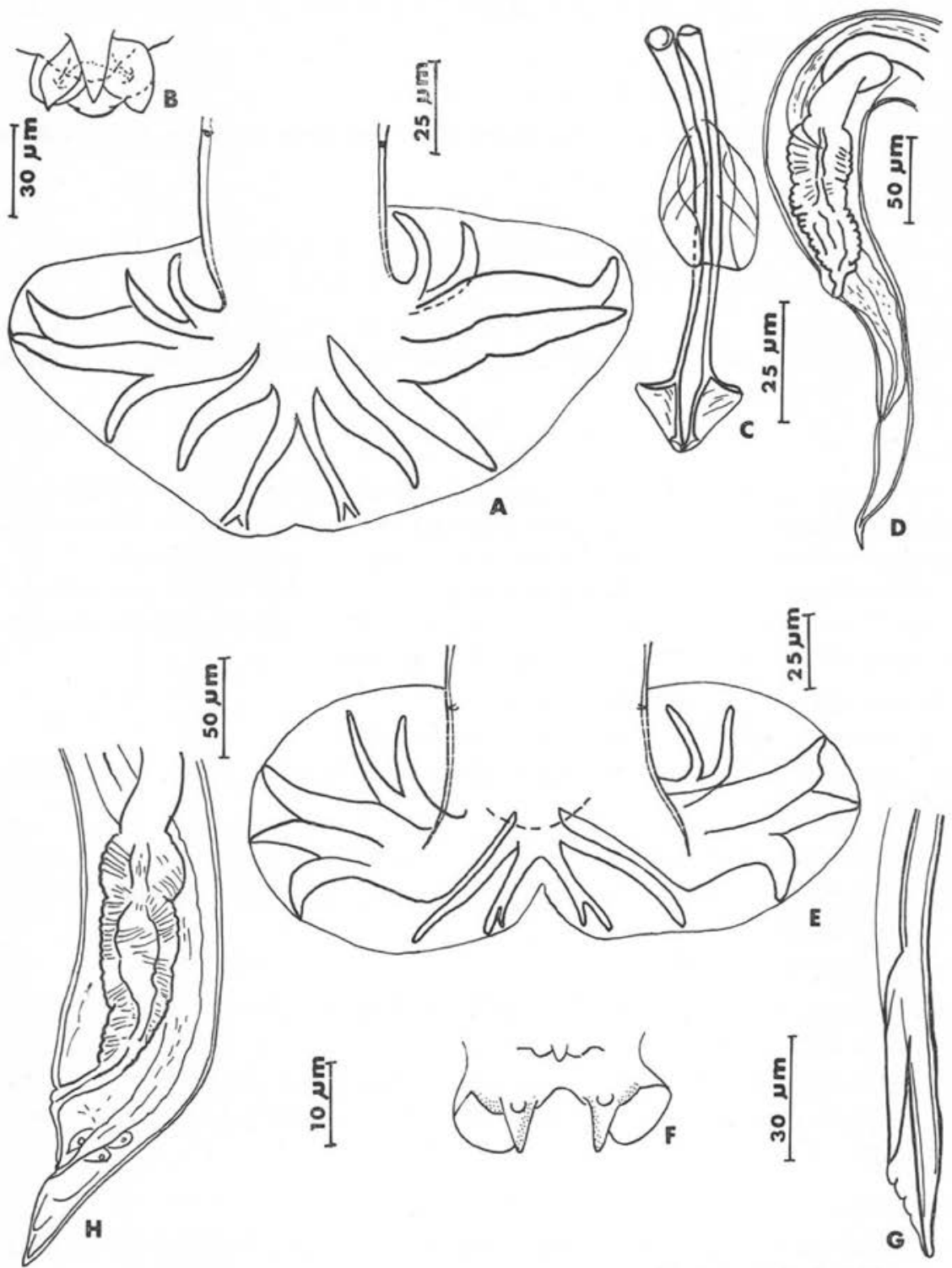

Fig. 5. - Paraheligmonelloides triangulus $\mathrm{n}$. sp. A, $\hat{\delta}$, bourse caudale, vue ventrale (dessin original retourné). B, $\hat{\delta}$, cône génital, vue ventrale. C, $\widehat{\sigma}$, spicules et gubernaculum, vue ventrale. $\mathrm{D}, \widehat{\sigma}$, extrémité postérieure vue latérale gauche. Paraheligmonelloides annandalei $\mathrm{n}$. sp. E, $\hat{\sigma}$, bourse caudale, vue ventrale (dessin original retourné). F, $\delta$, cône génital, vue ventrale. $\mathrm{G}, \widehat{\delta}$, pointe du spicule. $\mathrm{H}$, 우, extrémité postérieure, vue latérale gauche.

(D'après Ow Yang, 1967, in litt.) 
c) Paraheligmonelloides rajah n. sp.

(= Heligmonina (Heligmonoides) rajah Ow Yang, 1967 in litt.)

Hôte et origine géographique: Rattus rajah, de Malaisie.

Description (fig. 6).

- Synlophe : 16 arêtes cuticulaires au niveau du pore extréteur, dont 2 grandes arêtes ventrales gauches, 4 arêtes ventrales droites de taille décroissante du ventre vers la droite et 10 arêtes dorsales, dont les 2 droites sont les plus grandes.

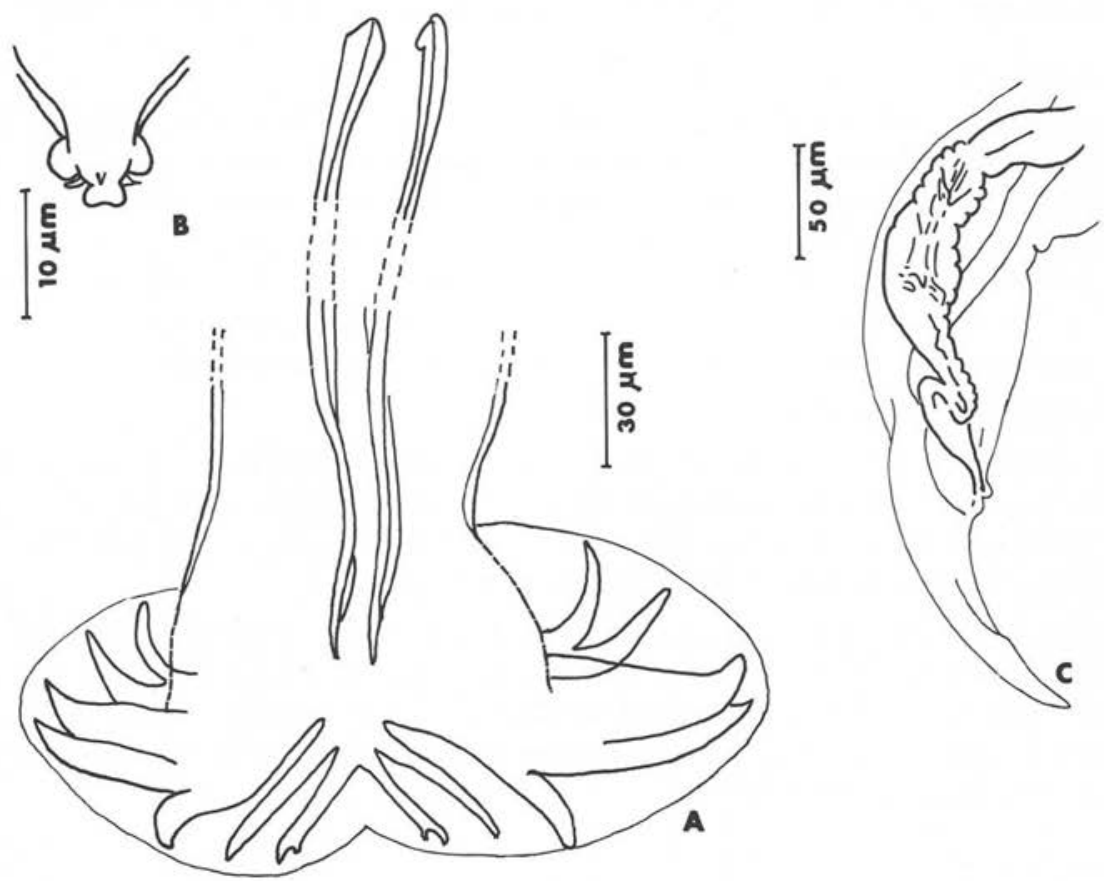

FIG. 6. - Paraheligmonelloides rajah n. sp. A, $\delta$, bourse caudale, vue ventrale (dessin original retourné). B, ô, cône génital, vue ventrale. C, 오, extrémité postérieure, vue latérale droite.

(D'après Ow Yang, 1967, in litt.)

\section{- Principales mensurations :}

Mâle : Longueur : 1,68 (1,62-2,22) mm sur 54 (50-57) $\mu \mathrm{m}$ de large. Spicules : 652 (469582) $\mu \mathrm{m}$. Gubernaculum : 60-70 $\mu \mathrm{m}$.

Femelle : Longueur : $1,96(1,69-2,27) \mathrm{mm}$ de long sur $54(50-57) \mu \mathrm{m}$. Vulve à 121 (106-162) $\mu \mathrm{m}$. Eufs hauts de $75(63-85) \mu \mathrm{m}$ sur 33 (26-43) $\mu \mathrm{m}$ de large. Queue : 41 (34-63) $\mu \mathrm{m}$. 


\section{Discussion}

Les spécimens ci-dessus sont très proches de Paraheligmonelloides annandalei. Ils s'en différencient par un synlophe ayant 2 arêtes dorsales supplémentaires, des spicules à pointe unique, une forme différente du gubernaculum et une position plus antérieure de la vulve.

6 - Genre Rattustrongylus gen. nov.

a) Rattustrongylus odontoconus n. gen., n. sp.

(= Longistriata odontoconus Ow Yang, 1967, in litt.)

Hôtes et origine géographique: Rattus sabanus (Matériel type), R. rajah, de Malaisie.

Description (fig. 7, A. à C, et fig. 11, E-F).

- Synlophe: 19 arêtes cuticulaires au milieu du corps dans les 2 sexes. Naissance des arêtes médianes et de la plupart des latérales en arrière de la vésicule céphalique. Les autres latérales naissent à différents niveaux le long des champs. Disparition des arêtes au niveau de la bourse caudale chez le $\widehat{\sigma}$ et de la vulve chez la

Axe d'orientation des arêtes de la ligne ventrale, ventrale-droite vers la ligne gauche. Arêtes dorsales gauches et ventrales droites les plus petites.

Dans la partie postérieure du corps, toutes les arêtes sont subégales.

- Principales mensurations :

Mâle : Longueur : $1,96(1,40-2,58) \mathrm{mm}$ sur 67 (57-85) $\mu \mathrm{m}$. Vésicule céphalique : 43 $(36-54) \mu \mathrm{m}$ sur $25(22-29) \mu \mathrm{m}$. Anneau nerveux et pore excréteur à $138(94-156) \mu \mathrm{m}$ et 181 (135-190) $\mu \mathrm{m}$ de l'apex. Deirides au même niveau. Esophage : 310 (270-391) $\mu \mathrm{m}$. Spicules : 333 (235-366) $\mu \mathrm{m}$. Gubernaculum : 43-51 $\mu \mathrm{m}$.

Femelle : Longueur : 2,34 (2,02-2,58) mm sur 67 (50-78) $\mu \mathrm{m}$. Vésicule céphalique : 42 $(37-46) \mu \mathrm{m}$ sur $27(22-34) \mu \mathrm{m}$. Anneau nerveux et pore excréteur à $125(94-153) \mu \mathrm{m}$ et $210(190-221) \mu \mathrm{m}$ de l'apex. Deirides au même niveau. Esophage : $320(284-355) \mu \mathrm{m}$. Vulve à 123 (111-136) $\mu \mathrm{m}$ de l'extrémité caudale. Eufs hauts de 67 (60-75) $\mu \mathrm{m}$ sur 38 (34-43) $\mu \mathrm{m}$. Queue : 35 (31-39) $\mu \mathrm{m}$.

\section{Discussion}

La disposition des côtes bursales est très semblable à celle des genres Paraheligmonelloides et Heligmonoides, avec cependant une asymétrie moins marquée des lobes bursaux, et des côtes ventrales relativement bien développées.

Par contre, le synlophe est beaucoup plus proche de celui d'un Paraheligmonelloides que d'un Heligmonoides, puisque les arêtes dorsales gauches sont peu développées.

Cependant, chez Paraheligmonelloides, au moins l'arête dorsale proche du champ latéral gauche est plus développée que les autres dorsales gauches ; chez les spécimens ci-dessus, toutes les arêtes dorsales gauches sont de même taille.

Ce caractère a une importante signification phylétique, qui sera analysée en détails ultérieurement. Schématiquement, on peut indiquer qu'à partir d'Orientos- 

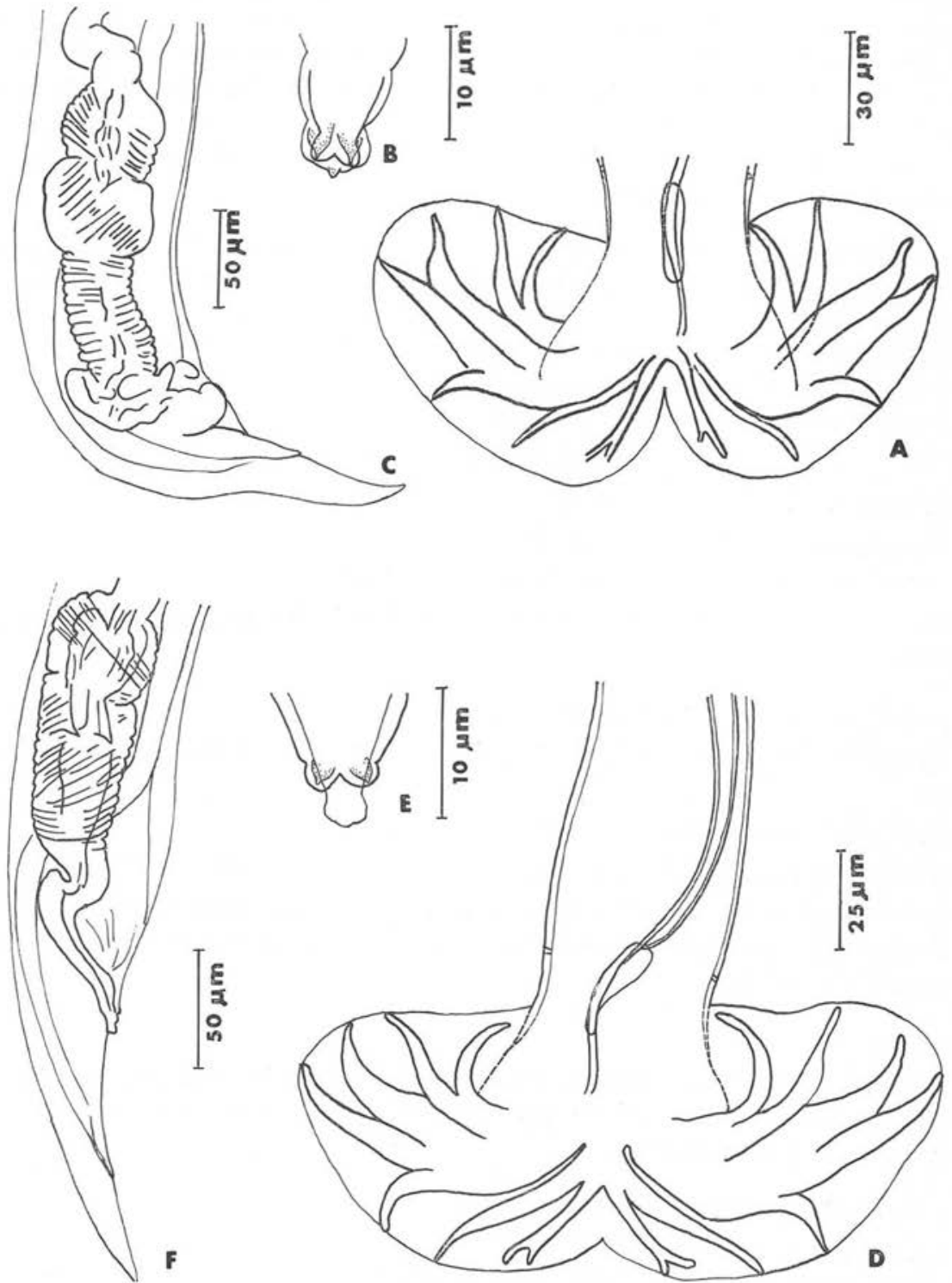

FIG. 7. - Rattustrongylus odontoconus n. gen., n. sp. A, ô., bourse caudale, vue ventrale (dessin original retourné). $\mathrm{B}, \hat{\delta}$, cône génital, vue ventrale. C, $\widehat{\delta}$, extrémité postérieure, vue latérale droite. Rattustrongylus rotundoconus n. sp. D, ô, bourse caudale, vue ventrale (dessin original retourné). E, ठ̊. cône génital, vue ventrale. F, O+, extrémité postérieure, vue latérale droite.

(D’après Ow Yang, 1967, in litt.) 
trongylus, deux lignées peuvent être différenciées. Dans un cas, les arêtes dorsales gauches tendent à augmenter de taille ; il en résulte un mode de fixation aboutissant à l'hypertrophie du lobe bursal gauche. Dans l'autre cas, les arêtes dorsales gauches tendent à diminuer de taille ; il en résulte un mode de fixation aboutissant à l'hypertrophie du lobe bursal droit.

Nous rangeons donc les spécimens ci-dessus dans un nouveau genre dont nous donnons la définition suivante :

Définition: Genre Rattustrongylus n. gen. : Heligmonellidae, Nippostrongylinae : Synlophe avec arêtes dorsales gauches égales entre elles et moins développées que les autres arêtes. Arêtes ventrales gauches et dorsales droites fortes.

Bourse caudale avec lobe gauche légèrement plus développé que le droit.

Côte dorsale divisée dans sa moitié antérieure.

Parasite de Rongeurs Muridae orientaux.

Espèce type : Rattustrongylus odontoconus n. gen., n. sp.

Autre espèce : $R$. rotundoconus n. sp.

b) Rattustrongylus rotundoconus n. sp.

(= Longistriata rotundoconus Ow Yang, 1967, in litt.)

Hôtes et origine géographique: Rattus rajah (Matériel type), Rattus sabanus, de Malaisie.

Description (fig. 7, D à F, et fig. 11, G).

- Synlophe : identique à celui de $R$. ratti avec 18 arêtes cuticulaires au milieu du corps.

\section{- Principales mensurations:}

Mâle : Longueur : $1,70(1,56-1,78) \mathrm{mm}$ sur $58(50-78) \mu \mathrm{m}$. Spicules : $275(225-315) \mu \mathrm{m}$. Femelle : longueur : $2,25(1,84-2,96) \mathrm{mm}$ sur $70(57-78) \mu \mathrm{m}$. Vulve à $121(94-136) \mu \mathrm{m}$ de l'extrémité caudale. Eufs hauts de $71(65-85) \mu \mathrm{m}$ sur $34(32-37) \mu \mathrm{m}$ de large. Queue : $32(26-43) \mu \mathrm{m}$.

Discussion

$R$. rotundoconus se distingue de l'espèce type par des côtes latérales plus épaisses, des côtes externo-dorsales qui atteignent le bord de la bourse caudale, l'absence de courbure de la queue chez la .

7 - Genre Sabanema gen. nov.

a) Sabanema sabana $\mathrm{n}$. sp.

(= Longistriata sabana Ow Yang, 1967, in litt.)

Hôtes et origine géographique: Rattus sabanus (Matériel type), $R$. argentiventer, $R$. canus, $R$. tiomanicus, $R$. rajah, Chiropodomys gliroides, de Malaisie.

Description (fig. 8, A à C, et fig. 11, H, I).

— Synlophe: $37\left(\widehat{o}^{3}\right), 40$ (ㅇ) arêtes cuticulaires au milieu du corps. Naissance des 


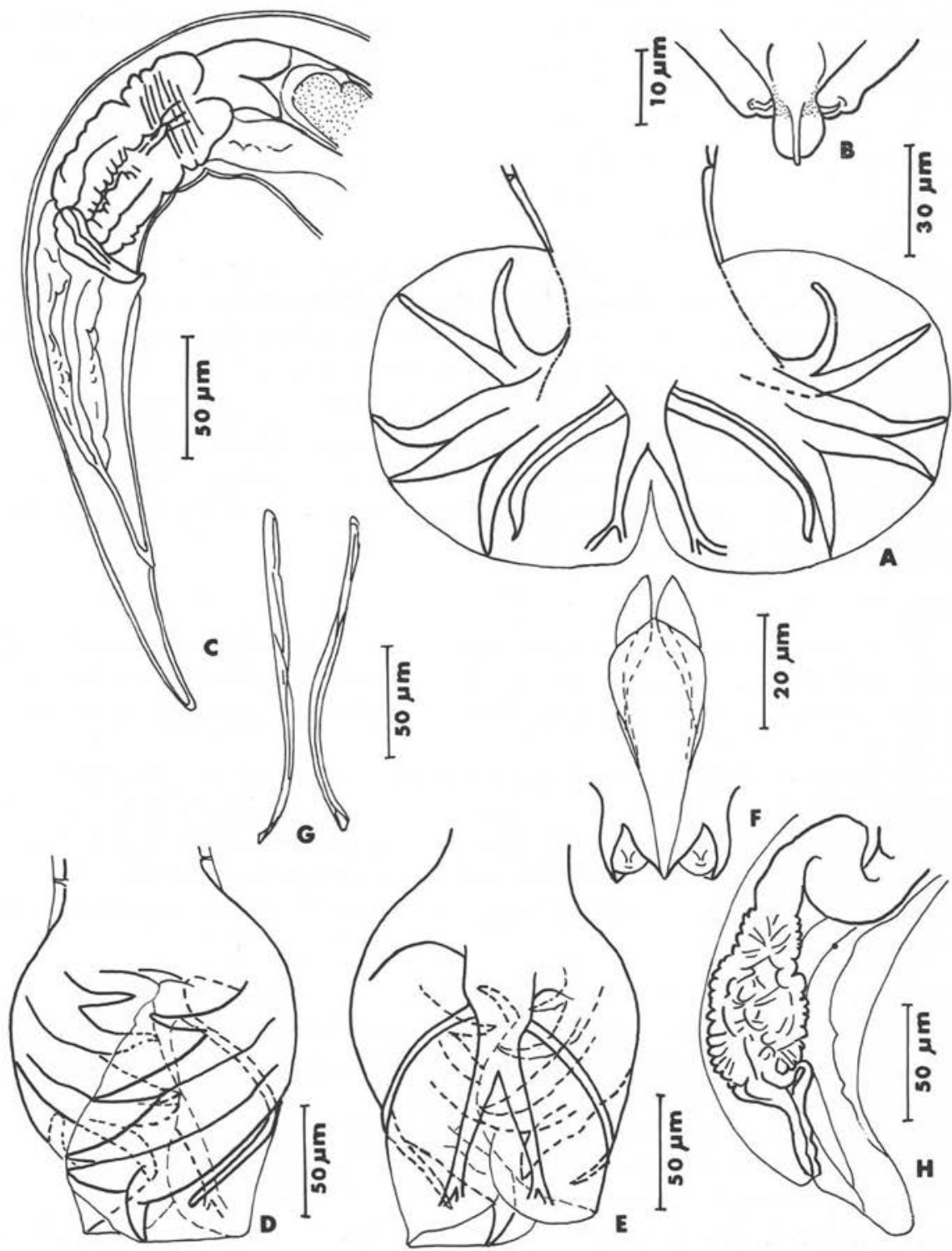

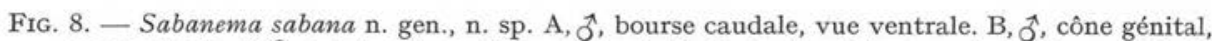
vue ventrale. C, ㅇ, extrémité postérieure, vue latérale droite. Sabanema kepongi $\mathrm{n}, \mathrm{sp} . \mathrm{D}, \hat{\mathrm{s}}$, bourse caudale, vue ventrale. E, $\widehat{\sigma}$, bourse caudale, vue dorsale. F, $\widehat{\delta}$, cône génital, vue ventrale. $\mathrm{G}, \widehat{\jmath}$, spicules. $\mathrm{H}$, 우, extrémité postérieure, vue latérale gauche.

(D'après Ow Yang, 1967, in litt.) 
arêtes médianes en arrière de la vésicule céphalique; des arêtes latérales soit en arrière de la vésicule céphalique, soit à différents niveaux le long des champs latéraux, soit par dédoublement d'arêtes. Disparition des arêtes au niveau de la bourse caudale chez le ô et de la vulve chez la 우.

Axe d'orientation : ligne ventrale-droite vers ligne gauche, dorsale-gauche. Les 4-5 arêtes droites et les 5-6 arêtes ventrales-gauches sont les plus fortes. Dans la partie postérieure du corps, toutes les arêtes sont sub-égales.

- Principales mensurations :

Mâle : Longueur : 2,49 (1,89-2,71) mm sur 88 (56-106) $\mu \mathrm{m}$. Vésicule céphalique : 50 $(48-56) \mu \mathrm{m}$ sur $30(27-34) \mu \mathrm{m}$. Anneau nerveux et pore excréteur à $153(142-170) \mu \mathrm{m}$ sur 206 (192-220) um de l'apex. Deirides au même niveau. Esophage : 354 (298391) $\mu \mathrm{m}$. Spicules : $260(240-272) \mu \mathrm{m}$. Gubernaculum : $50 \mu \mathrm{m}$.

Femelle : Longueur : 5,54 (2,77-10,8) mm sur 89 (71-107) $\mu$ m. Vésicule céphalique : 54 $(43-60) \mu \mathrm{m}$ sur $30(26-36) \mu \mathrm{m}$. Anneau nerveux et pore excréteur à 151 (128-192) $\mu \mathrm{m}$ et $192(149-256) \mu \mathrm{m}$ de l'apex. Deirides au même niveau. Esophage : 349 (262-390) $\mu \mathrm{m}$. Vulve à 161 (93-248) $\mu \mathrm{m}$ de l'extrémité caudale. 15 œufs hauts de 65 (60-68) $\mu \mathrm{m}$ sur 35 (34-39) $\mu \mathrm{m}$. Queue : $51(27-68) \mu \mathrm{m}$.

\section{Discussion}

Le genre le plus proche est Rattustrongylus. La disposition des côtes bursales (côtes ventrales en V fermé, asymétrie de la bourse caudale), le synlophe (arêtes dorsales gauches subégales et petites) sont des caractères qui montrent la parenté des 2 genres.

Les spécimens ci-dessus s'en éloignent par une côte dorsale plus développée, avec un tronc commun à la dorsale et aux côtes 8 plus long, et surtout par un plus grand nombre d'arêtes cuticulaires (plus de 25 au niveau du pore excréteur, plus de 35 au milieu du corps pour les espèces dont le synlophe est connu à ce niveau.

Nous classons donc ces spécimens dans un nouveau genre, que nous nommons Sabanema gen. nov.

Définition: Genre Sabanema n. gen. : Heligmonellidae, Nippostrongylinae :

Synlophe avec nombreuses arêtes (plus de 25 au niveau du pore excréteur). Arêtes dorsales gauches et ventrales droites subégales et plus petites que les autres arêtes. Bourse caudale avec lobe gauche plus développé.

Côte dorsale assez longue, divisée au moins aux deux tiers de sa hauteur.

Parasite de Rongeurs Muridae orientaux.

Espèce type : Sabanema sabana n. sp.

Autres espèces : S. kepongi n. sp. ; S. macrovula n. sp. ; S. montana n. sp. ; S. longispicularis n. sp.

b) Sabanema kepongi n. sp.

(= Longistriata kepongi Ow Yang, 1967, in litt.)

Hôtes et origine géographique : Rattus cremoriventer (Matériel type), $R$. annandalei, $R$. sabanus, Chiropodomys gliroides, de Malaisie; $R$. fluvescens, de Thaïlande. 
Description (fig. 8, D à H, et fig. 11-J).

- Synlophe: identique à celui de l'espèce précédente avec 32 à 34 arêtes au niveau du pore excréteur ( $\widehat{o}$ et \%), et 37 au milieu du corps (ㅇ).

- Principales mensurations :

Mâle : Longueur : 2,97 (2,48-3,39) mm sur 97 (71-121) $\mu \mathrm{m}$. Spicules : $139(127-145) \mu \mathrm{m}$. Gubernaculum : $40 \mu \mathrm{m}$.

Femelle : Longueur : $3,65(2,98-4,31) \mathrm{mm}$ sur $119(99-128) \mu \mathrm{m}$. Vulve à $38(34-49) \mu \mathrm{m}$ de l'extrémité caudale. Eufs hauts de 61 (53-68) $\mu \mathrm{m}$ sur 31 (26-34) $\mu \mathrm{m}$. Queue : 19 $(14-20) \mu \mathrm{m}$.

\section{Discussion}

Les spécimens ci-dessus sont très proches de $S$. sabana, avec un synlophe identique et une disposition semblable des côtes bursales. Ils s'en distinguent chez le đ̊ par l'éloignement des extrémités des côtes 6 et 8 , par des spicules bifurqués à leur extrémité, et, chez la 우, par une queue courte, épaisse et arrondie, ainsi qu'un très grand nombre d'œufs.

c) Sabanema macrovulva n. sp.

(= Longistriata macrovulva Ow Yang, 1967, in litt.).

Hôte et origine géographique: Rattus sabanus, de Malaisie.

Description (fig. 9, $A$ à $D$ ).

- Synlophe : 38 à 42 arêtes cuticulaires au niveau du pore excréteur. Arêtes dorsales gauches petites et sub-égales.

- Principales mensurations :

Mâle : longueur : 2,78 (2,51-3,51) mm sur 71 (57-99) $\mu \mathrm{m}$. Spicules : $332(281-357) \mu \mathrm{m}$. Gubernaculum : $34 \mu \mathrm{m}$.

Femelle : Longueur : 3,94 (3,67-4,78) mm sur 95 (78-107) $\mu \mathrm{m}$ de large. Vulve à 112 $(102-128) \mu \mathrm{m}$ de l'extrémité caudale. 18 œufs hauts de $66(60-71) \mu \mathrm{m}$ sur $38(34-43) \mu \mathrm{m}$.

\section{Discussion}

Par son nombre élevé d'arêtes cuticulaires, dont les dorsales gauches sont petites et sub-égales, et par la disposition de ses côtes bursales, l'espèce peut aisément être rangée dans le genre Sabanema.

Elle se distingue des 2 autres espèces par une asymétrie plus forte de la bourse caudale et par des spicules beaucoup plus longs pour une taille comparable.

d) Sabanema longispicularis n. sp.

(= Pseudoheligmostrongylus longispicularis Ow Yang, 1967, in litt.)

Hôtes et origine géographique: Rattus sabanus (Matériel type), R. rajah, de Malaisie.

Description (fig. 9, E à I).

- Synlophe : Identique à celui de l'espèce type avec 26-30 arêtes au niveau du pore excréteur. 


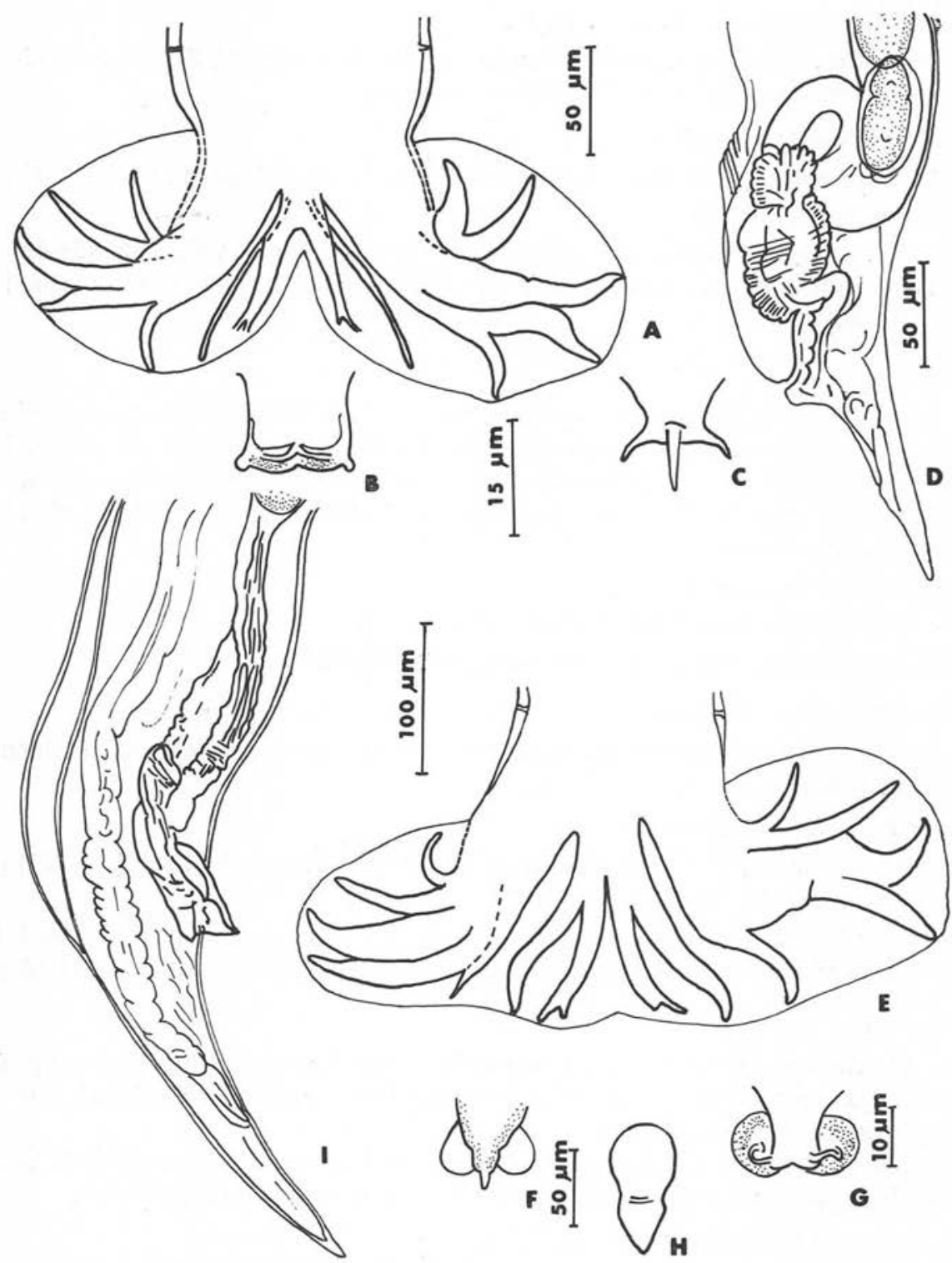

FIG. 9. - Sabanema macrovulva $\mathrm{n}$. sp. A, §̂. bourse caudale, vue ventrale (dessin original retourné). $\mathrm{B}, \widehat{O}$, lèvre dorsale du cône génital, vue ventrale. C, ô., lèvre ventrale du cône génital, vue ventrale. D, 오, extrémité postérieure, vue latérale gauche. Sabanema longispicularis n. $\mathrm{sp}$. $\mathrm{E}, \hat{\sigma}$, bourse caudale, vue ventrale (dessin original retourné). $\mathrm{F}, \hat{\sigma}$, lèvre ventrale du cône génital, vue ventrale. $\mathrm{G}$, lèvre dorsale du cône génital, vue dorsale. $\mathrm{H}$, languette vulvaire, vue ventrale. I, ㅇ, extrémité postérieure, vue latérale droite.

(D’après Ow Yang, 1967, in litt.) 


\section{- Principales mensurations :}

Mâle : Longueur : 2,52 (2,25-2,67) mm sur $71(64-92) \mu \mathrm{m}$. Spicules : $1,05(0,87-1,16)$ mm. Gubernaculum : $94 \mu \mathrm{m}$.

Femelle : Longueur : 3,41 (2,91-3,96) mm sur 97 (71-135) $\mu \mathrm{m}$. Vulve à 254 (204264) $\mu \mathrm{m} .9$ œufs hauts de $70(65-77) \mu \mathrm{m}$ sur 34 (29-41) $\mu \mathrm{m}$. Queue : $90(68-111) \mu \mathrm{m}$.

\section{Discussion}

Le grand nombre d'arêtes cuticulaires et les caractères bursaux permettent de classer les spécimens ci-dessus dans le genre Sabanema.

L'espèce peut se différencier par l'allongement transversal de la bourse caudale, par des spicules atteignant plus du tiers de la longueur du corps et par la présence d'une languette prévulvaire chez la femelle.

e) Sabanema montana n. sp.

(= Heligmonina (Neoheligmonina) montana Ow Yang, 1967, in litt.)

Hôte et origine géographique: Rattus alticola, de Malaisie.

Description (fig. 10).

- Synlophe: 37 à 39 arêtes cuticulaires au niveau du pore excréteur. Arêtes dorsales gauches plus petites que les droites.
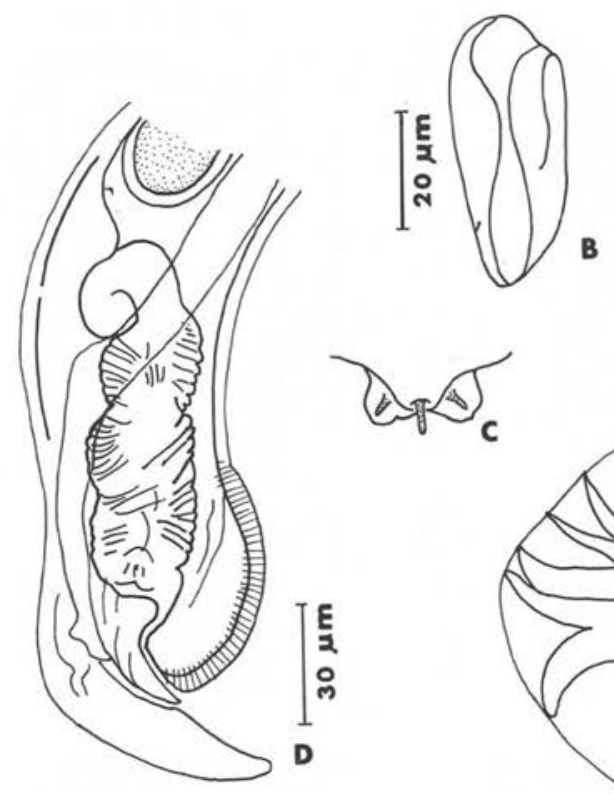

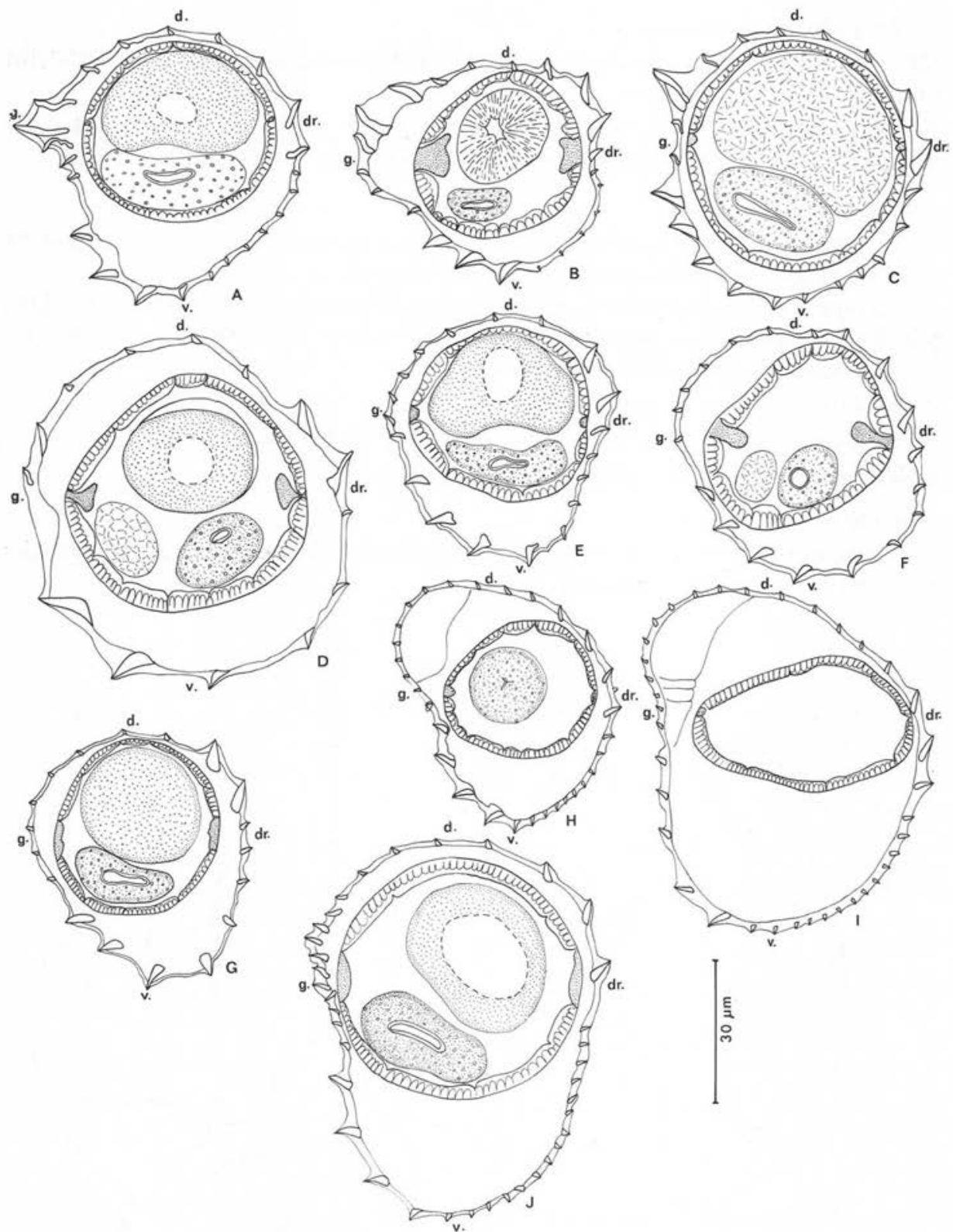

FIG. II. - Coupes transversales au milieu du corps, sauf la fig. $\mathrm{H}$ faite au niveau du pore excréteur. Heligmonoides bulbosus n. sp. A, 우, B, ㅈ․ Paraheligmonelloides triangulus $\mathrm{n} . \mathrm{sp} . \mathrm{C}, ㅇ$

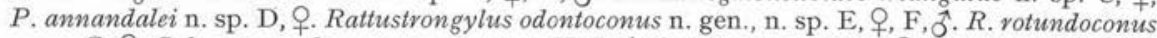
n. sp. G, ㅇ. Sabanema sabana n. gen., n. sp. H, I, ô. S. kepongi n. sp. J, ㅇ․

(D'après les paratypes de Malaisie). 


\section{- Principales mensurations:}

Mâle : Longueur : $2,11(1,90-2,59)$ mm sur 80 (64-99) $\mu \mathrm{m}$. Spicules : $120(111-136) \mu \mathrm{m}$. Gubernaculum : $36 \mu \mathrm{m}$.

Femelle : Longueur : $2,35(2,02-2,72) \mathrm{mm}$ sur $73(64-92) \mu \mathrm{m}$. Vulve à $52(46-60) \mu \mathrm{m}$ de l'extrémité caudale. 6 œufs hauts de 65 (60-68) $\mu \mathrm{m}$ sur $32(29-34) \mu \mathrm{m}$. Queue : 33 (29-34) $\mu \mathrm{m}$.

\section{Discussion}

Par les caractères de son synlophe (nombreuses arêtes cuticulaires, dont les dorsales gauches et les ventrales sont petites), l'espèce peut aisément être rangée dans le genre Sabanema.

Elle s'éloigne des autres espèces par des côtes 8 épaisses et par le grand développement des côtes 5 et 6 gauches.

\section{BIBLIOGRAPHIE}

Baylis H. A. : On a collection of Nematodes from Nigerian mammals (chiefly Rodents). Parasitology, 1928, 20, 280-304.

Chabaud A. G., Desset M.-C. : Nippostrongylus rauschi $\mathrm{n}$. sp. Nématode parasite de Dermoptères et considérations sur $N$. brasiliensis parasite cosmopolite des Rats domestiques. Ann. Parasitol. Hum. Comp., 1966, 4I, 243-249.

Durette-Desset M.-C. : Les systemes d'arêtes cuticulaires chez les Nématodes Héligmosomes. II. Étude de cinq Longistriata, parasites de Rongeurs africains. Cah. La Maboké, 1966, 4, I $20-140$.

Durette-Desset M.-C. : Nématodes héligmosomes d'Amérique du Sud. I. Description de deux nouvelles espèces : Stilestrongylus freitasi, parasite de Zygodontomys lasiurus, et Viannella lenti, parasite de Galea spixi. Bull. Mus. Natn. Hist. Nat., 1968, 2e sér., 40, 403-412.

DuRETTE-DESSET M.-C. : Caractères primitifs de certains Héligmosomes, parasites de Muridés et de Cricétidés orientaux. Définition d'Orientostrongylus n. gen. Ann. Parasitol. Hum. Comp., 1970, 45, 829-837.

Duretre-Desset M.-C. : Essai de classification des Nématodes Héligmosomes. Corrélation avec la paléobiogéographie des hôtes. Mém. Mus. Natn. Hist. Nat., 1971, nlle sér., sér. A, Zool., 49, $126 \mathrm{p}$.

Durette-Desset M.-C. : Brevistriatinae (Nematoda - Heligmosomidae). I. Compléments morphologiques à l'étude d'espèces connues. Bull. Mus. Natn. Hist. Nat., 1976, $3^{\text {e }}$ sér., $\mathrm{n}^{0} 3^{88}$, Zool. $270,685-692$.

Durette-Desset M.-C., Chabaud A. G. : Description d'un nouveau nématode héligmosome parasite d'un écureuil volant. Bull. Soc. zool. Fr., 1967, 92, 227-233.

DuretTe-Desset M.-C., Lim Boo LiAT : Description de deux nouvelles espèces du genre Orientostrongylus Durette-Desset, 1971. (Nématode, Héligmosome) chez un Tupaiiné et un Petauristiné de Malaisie. Ann. Parasitol., Hum. Comp., 1974, 49, 669-675.

Durette-Desset M.-C., Chabaud A. G. : Sur trois Nématodes Trichostrongylides parasites de Tupaiidae. Ann. Parasitol. Hum. Comp., 1975, so, I73-185.

DuretTe-DesSET M.-C., Krishnasamy M. : Brevistriatinae (Nematoda : Heligmosomidae). III. Description de Fissicauda n. gen. et de Kuala n. gen. parasites de petits Mammifères malais. Bull. Mus. Natn. Hist. Nat., 1976, $3^{\text {e }}$ sér., no 388, Zool. 270, 697-710.

Fukumoto S., KamiYa M., Suzuki H., : Three Trichostrongylid nematodes from the red rock rabbits Pronolagus sp. in Kenya. Jap. J. Vet. Res., 1980, 28, 129-136.

LANE C. : Some Strongylata. Parasitology, I923, Is, 348-364.

Lubimov M. P. : Pithecostrongylus satyri n.g., n. sp., trouvé dans l'intestin d'un Orang-Outang. Ann. Parasitol., Hum. Comp., 1930, 8, 51-56.

OhbaYASHi M., KAmiYA M. : Studies on the parasite fauna of Thailand. II. Three nematode species of the genus Orientostrongylus Durette-Desset, 1970. Jap. J. Vet. Res., 1980, 28, 7-II.

ORTLEPP R. J. : South African helminths. Part VI. Some helminths, chiefly from rodents. Onderstepoort J. Vet. Sci. Am. Ind., 1939, I2, 75-101.

Ow YANG C. K. : A new species of Hooperstronsylus Lie and Ow Yang (Trichostrongylidae, Nematoda) from the intestine of Squirrels in Malaya with some remarks on the genus. Zool. Anz., $1965,174,323-328$. 
Ow YANG C. K. : Systematics and life history studies on nematodes of Malayan rodents. Thèse Ph. D., Université de Londres, 1967.

Ow Yang C. K. : Studies on the nematode parasites of Malaysian rodents. I. Rhabdiasidea, Trichuridea and Oxyuridea. J. Helminthol., I971, 46, 93-109.

Schacher J. F., CheONG C. H. : Malaysian parasites XLVII. Nematode parasites of three common house rat species in Malaya, with notes on Rictularia tani Hoeppli, 1929. Studies Inst. Med. Research, Feder. Malaya, 1960, 29, 209-216.

Schmidt G. D., Myers B. J., Kuntz R. E. : Nematode parasites of Oceanica. I. Brevistriata sundasciuri sp. n. and Calypsostrongylus ogdeni gen. et sp. n. (Heligmosomatidae : Longistriatinae) from squirrels of Palawan and Taiwan. J. Parasitol., 1967, 53,613-6r7.

SingH M., CheONG C. H. : On a collection of nematodes parasites from Malayan rats. South-east Asian J. Trop. Med. Publ. Hlth, I97I, 2, 516-522.

Supperer R., Kutzer E. : Zwei neue Trichostrongyliden aus dem Flaggenhörnchen, Brevistriata callosciuri nov. spec. und Pithecostrongylus univesicula nov. sp. Z.. Parasitenka, I963, $23,1 \mathrm{I}-15$.

TENoRA F. : Parasitic nematodes of certain rodents from Afghanistan. Vestnik Cs. spol. Zool., I969, 33, I74-192.

Travassos L. : Trichostrongylideos brazileiros (III nota previa). Brazil. Med., I9I4, 28, 325-327.

YEH L. S. : A new bursate nematode Hepatojarakus malayae gen. et sp. nov. from the liver of Rattus rattus jarak (Bonhote) on Pulan Jarak, Straits of Malacca. J. Helminthol., 1955, 29, 44-58. 